\title{
Trayectorias de las editoras profesionales Del fin del siglo XIX en Chile ${ }^{1}$
}

\section{Trajectories of Professional Women Editors from the Late $19^{\text {th }}$ Century in Chile}

\author{
CLAUDIA MONTERO \\ Universidad de Valparaíso, Instituto de Historia y Ciencias Sociales. \\ Correo electrónico: claudia.montero@uv.cl
}

En este trabajo se revisan las biografías de mujeres editoras de medios de prensa en Chile en el fin del siglo XIX. A partir de sus trayectorias vitales se problematiza la relación de las mujeres con la profesionalización y el ingreso al campo cultural en un momento crítico para la conformación de este. Se establece una definición de editora considerando la transformación de la misma de acuerdo al contexto histórico. Se demuestra que el ser editora fue una forma de ingreso al campo cultural, que les permitió a las mujeres salvar normas de género. La especialización como editoras las convirtió en profesionales a través de estrategias de autoformación y ocupando las escasas posibilidades de educación formal con que contaban en ese momento.

Palabras claves: editoras, campo cultural, mujeres, Chile, siglo XIX.

This paper studies biographies of paper's women editors in Chile at the end of the $19^{\text {th }}$ century. Considering their trajectories, I analyse the relationship between women and professionalization and the access to the cultural field in a critical moment for its conformation. A definition of women editor is made which considers a transformation according to historical context. It is demonstrated that to be an editor was a way to access to cultural field. This permits women manage gender norms. The specialization as editors made them professionals. They developed strategies of self-education taking advantage of the few possibilities of formal education available in that moment.

Key words: Women editors, cultural field, women, Chile, 19 Century.

\footnotetext{
${ }^{1}$ Este trabajo forma parte de la investigación Fondecyt Iniciación № 11160361: “Tomarse la palabra: mujeres editoras en el campo cultural Chileno 1875-1930”. Investigadora Responsable: Dra. Claudia Montero.
} 


\section{INTRODUCCIÓN}

El ingreso de las mujeres al campo cultural chileno ha sido estudiado considerando a intelectuales, escritoras, traductoras, antologistas, críticas y redactoras. Este fenómeno se enmarca en el cambio modernizador de fin del siglo XIX que posibilita condiciones para que las mujeres demanden un lugar en el campo cultural. Sin embargo, la complejidad del fenómeno de transformación del cambio de siglo hace que existan lugares de intervención aún inexplorados. Es el caso de las editoras, quienes a pesar de que existen registros desde fin del siglo XIX de mujeres como propietarias, directoras, administradoras u otras labores asociadas al manejo de medios de prensa, aún no han sido estudiadas.

La especificidad de la acción editorial resulta necesaria de analizar ya que ilumina el nudo problemático de mujeres y profesionalización. En momentos en que se abrían tímidamente las puertas de acceso para una educación formal universitaria para las mujeres, la edición fue un oficio que les permitió no sólo ingresar al campo cultural, sino adquirir una profesión que les permitió subsistencia y desarrollo personal.

El objetivo de este trabajo es revisar las trayectorias de mujeres editoras de medios en Chile en la última parte del siglo XIX. Específicamente, se establecen las condiciones que posibilitan el ingreso de las mujeres al campo cultural, la definición de editora y su relación con la profesionalización. Finalmente se revisan las trayectorias vitales de las editoras para mostrar que las trayectorias vitales de las mujeres que se dedicaron a la edición evidenciaron las dificultades que tuvieron las mujeres para ingresar al campo cultural, teniendo que desarrollar estrategias de autoformación además de utilizar las escuálidas posibilidades de profesionalización con que contaban en ese momento.

\section{Mujeres en el Campo cultural a fin del siglo XIX}

La acción de las editoras se encuentra en el contexto de la inserción de las mujeres en el mundo de las letras en el proceso de modernización latinoamericano. Este fenómeno forma parte de la emergencia de nuevos sujetos sociales que se tomaron la palabra en la ciudad modernizada (1880-1920) definida por Ángel Rama (1998). A pesar que este autor no se refiere a las mujeres en particular, es útil ya que plantea que son intelectuales que respondieron a nuevas características y que desafiaron al poder, combatiendo los privilegios de la ciudad letrada para dar paso a nuevos grupos sociales (Rama 62). El caso de las mujeres es particular, ya que su desafío incluyó la transgresión de la norma de género que les impedía la expresión pública. Al respecto, Batticuore (1994) da cuenta de cómo la escritura femenina era consideraba una afrenta contra el honor de la mujer que osara publicar. La incursión de las mujeres en el campo cultural implica el peligro de que, al mismo momento de hacer el ingreso, podrían perder el derecho a la palabra (Irigaray 1985). Considerando las ideas de Irigaray, esta contradicción también forma parte del ser editora, quien frente al riesgo de perder el derecho al habla crea un lenguaje propio, que en este caso es la edición. 
El momento histórico en el que nos situamos es clave por las transformaciones sociales, políticas y culturales. Según Rama, en América Latina se dio cuerpo a una gramática propia que articuló orden y dignidad lingüística. De acuerdo con ello, Soledad Falabella (2012) plantea que se reconoció la polifonía "con potencia democratizadora, gesto clave para la legitimación del espacio letrado tensionado y múltiple en su heterogeneidad" (125). Con ello se abrió la norma letrada a sujetos antes excluidos entre los que se contaban las mujeres. La letra fue la palanca de ascenso social en un contexto donde los Estados crecieron y asumieron proyectos de educación pública (Rama 62). Para Claudia Darrigrandi (2014), los intelectuales del cambio modernizador, dentro de ellos las editoras, poseían una nueva sensibilidad que les permitía leer los procesos sociales desde otra perspectiva, para ver otros fenómenos y evidenciar otros significados (Darrigrandi 20). Todo ello se expresaba en las decisiones editoriales de los medios de los que estaban a cargo.

La acción editorial de las mujeres, consideraba además de la sensibilidad antes descrita, la experiencia como sujetos excluidos por el género. Esta permite develar una perspectiva antes no reconocida, muchas veces negada y que naturalizaba la exclusión. Recogiendo la idea de Darrigrandi que para el estudio de lo urbano plantea cómo éstos sujetos sociales "ven otras ciudades" (20), en este caso las editoras serían capaces de ver otras realidades, otras formas de concebir lo cultural y literario. Esto porque, como plantea Mary Louise Pratt (1993), las mujeres se definen en diálogo con las ideas hegemónicas estableciendo una línea de heterogeneidad dada por el género sexual. Así se reconocen en un lugar marginal y como mediadoras, en el que "su autorización se construye a partir de la misma ambigüedad de su ciudadanía" (Pratt 54). Las mujeres, dice Pratt, en tanto escritoras, comentadoras, traductoras (aquí sumaríamos editoras) enfocaron su trabajo cultural en prácticas de mediación transnacional. Es decir, que eran capaces de ver más allá, tensando ideas y supuestos.

Periódicos y revistas fueron el mecanismo de difusión de las voces que se autonomizaron de la ciudad letrada. Como dice Darrigrandi, éstos encarnaron en papel el cambio modernizador que implicó el desarrollo de la cultura de masa, la especialización del oficio del escritor y la profesionalización de la prensa (Darrigrandi 20). Por lo tanto, la edición y producción de estos medios implicó dar cuerpo a voces críticas de una sociedad en cambio. Según Juan Poblete (2006), el periódico fue un "aparato cultural nuevo y crucial en las dinámicas discursivas del siglo XIX: circulación lectores y formas de lectura, tipos de discursos y formatos, autoconcepción y objetivos en relación a un público lector, comunidades discursivas, jerarquías culturales".

Las décadas de 1870 a 1890 representan un momento particular. Es muy complejo hablar de una industria editorial, de la profesionalización del escritor o de la editora a la que nos referimos. Sin embargo, es un momento clave definido por Julio Ramos (2009), con reminiscencias de la ciudad letrada de Rama y con marcas del orden oligárquico (Brunner y Catalán 1985). Según Ramos, hasta 1880 letra y acción pública seguían sin representar una relación problemática. La escritura continuaba manteniendo una función civilizatoria, fenómeno que cambió con la consolidación de los Estados en las dos últimas décadas del 
siglo XIX. Sólo a partir del fin de siglo se puede comenzar a hablar de un surgimiento paulatino de una esfera específica para la política y otra para la república de las letras. Las ideas de Ramos nos ayudan a entender este período como un momento de desarrollo de una cultura impresa donde escribir seguía siendo un acto político. Es allí donde la acción de las mujeres en la letra cobraría ribetes de transgresión. Ana Traverso (2012) plantea la escritura de las mujeres de inicio de siglo como un ejercicio para significarse ellas mismas como críticas de los cambios sociales de la época, lo que incluye un cuestionamiento social al sometimiento de las mujeres. La escritura como acto político según Ramos, implica que no fue un proceso reducido al cambio y especialización del empleo como el de periodista, sino un fenómeno con muchas aristas donde las editoras jugarían una parte.

\section{LA EDITORA Y LA PROFESIONALIZACIÓN DE LAS MUJERES}

Los análisis sobre la labor editorial no han discutido la definición del editor/a. Esto supone un problema ya que en el mundo del impreso existen muchos tipos de éstos: libros, periódicos, revistas (y su innumerable variedad), volantes, etc. Cada uno de ellos implica una labor editorial específica, con labores diferenciadas de acuerdo al producto impreso que se produce. En general existen más estudios que abordan a editores o editoras relacionados al mundo del libro. Estudios sobre editoras de prensa existen menos. Sin embargo, en todos los casos, se confirma que es una labor femenina invisibilizada por la historia del libro, de la prensa y la cultura escrita. Sin embargo, supone una dimensión de la acción femenina en el espacio público fundamental para comprender su construcción.

La definición que proponemos de editora es amplia. Abarca cualquier relación con el mundo de producción de medios de prensa: dueña de imprenta, de medios, dueña de periódico o revista, fundadora, directora o editora en términos de quien decide qué contenidos se incluyen en una publicación periódica. La amplitud de la definición nos permite dar cuenta de la complejidad del fenómeno y de las múltiples dimensiones y lugares desde donde se toman decisiones al momento de publicar un medio. Además, depende de los objetivos al momento de asumir la función editorial frente a un medio. Por lo tanto, podemos afirmar que esta acción es plástica, se configura y transforma dependiendo de los factores antes mencionados.

Dentro del estado del arte sobre la acción de las mujeres en la prensa en Chile, esta se registra desde la segunda mitad del siglo XIX. Fue un movimiento moderado y no exento de polémica. El formato periódico o revista fue utilizado por las mujeres para camuflar su acción como sujetos con opinión en un medio adverso (Montero 2016). Dentro de este movimiento, encontramos la figura definida por Carol Arcos (2014) como "la publicista". Ésta representó la consolidación de las mujeres como sujetos y su asunción como autoras fundando un nuevo rol social femenino que iba más allá de la maternidad. "La publicista" emitió opinión y diversificó sus formas discursivas. La misma situación representa "la editora" definida también por Arcos, como una figura que ingresó al campo cultural a 
través de revistas ilustradas, desarrolladas como empresas comerciales. Otros estudios sobre mujeres en la prensa en Chile consideran aspectos generales sobre su acción de producción de medios, sin problematizar necesariamente el concepto de editora.

La diversidad de publicaciones periódicas que se han registrado bajo la responsabilidad de mujeres permite visualizar la flexibilidad de la que hablamos al referirnos a la editora. Podemos verla en las formas de circulación de las publicaciones: comercial o no comercial; el formato: periódicos o revistas; los temas: prensa política, de literatura, cine, infantil o revistas femeninas. Podríamos pensar la figura de la editora como un espacio o lugar de enunciación marginal dada las normas de género que le permite configurarse como profesional.

La profesionalización de las mujeres en las letras es un tema que se ha abordado, pero sin discutir profundamente qué se entiende por ello. Hay múltiples criterios que se han usado para caracterizar a una mujer profesional de este ámbito. El más presente en las escritoras latinoamericanas es el relacionado con ser una intelectual. Sin embargo, esto nos lleva a una nueva definición: la de intelectual. Para ello se han establecido indicadores como el reconocimiento, la trayectoria de escritura, la cantidad de publicaciones, desde qué edad comenzó a publicar, quién fue su figura de autorización y la recepción de la crítica. Otro indicador es el sostenimiento económico a partir de su labor de escritura, es decir, si recibieron un sueldo por sus textos o por sus productos editoriales o si vivían de ello.

Hablar de la profesionalización de las mujeres requiere comprender que ésta significa cosas distintas o tiene diversas características considerando el momento histórico en el que situemos el problema. De tal forma no es lo mismo hablar de la profesionalización de las escritoras a fin del siglo XIX que a inicios del XX.

Las discusiones contemporáneas sobre profesionalización plantean una idea amplia centrada en las trayectorias de las personas (González-Leandri 2006: 335). Se considera el camino vital de las personas para desarrollar una carrera, independiente de su formación, educación y trabajos en los que se han desempeñado (Brown 2018). Estas ideas permiten afirmar que la acción de las editoras, en la medida que fue una práctica al producir los medios de prensa, puede ser considerada como una profesión propiamente tal.

Un primer elemento es la cuestión de la educación. Dado el contexto histórico de inicios de un sistema educativo nacional, hay heterogeneidad en la formación de las mujeres a las que nos referimos. Algunas recibieron educación formal, en escuelas primarias o secundarias; otras, educación no formal en sus casas; ninguna recibió un título universitario para desempeñarse como escritora o editora. Las perspectivas nuevas sobre profesionalización establecen que ésta se define como el camino de una persona desempeñando una labor considerando cualquier tipo de capacitación o tipo de educación. De tal forma, no es necesario que estas hubiesen tenido un título profesional, ya que por una parte no existían a fin del siglo XIX en Chile condiciones reales para las mujeres para que lo obtuvieran, y porque sus decisiones vitales y formación no formal e informal (es decir lo que aprendieron haciendo) las hizo profesionales.

Un camino común entre las editoras de fin del XIX fue la docencia. Esta fue una puerta de entrada a la profesionalización abierta por la ventana de oportunidad para las 
mujeres dada por el aumento de la demanda de profesoras para satisfacer la política estatal de abrir escuelas públicas para nińas. Se accedía por dos caminos: uno con reconocimiento de hecho y otro con titulación. La primera forma correspondía a la ayudantía. Es decir, en tanto estudiante adelantada, ser promovida para ejercer labores de enseñanza (Egańa, Salinas y Nuñez 2000: 99). La segunda, fue la titulación brindada por las escuelas normales femeninas abiertas a partir de 1853. Las editoras que se titularon de preceptoras o fueron ayudantes, no sólo fueron profesionales de la enseñanza, sino que a partir de allí continuaron el camino de la edición en la que se alzaron como profesionales editoriales. La investigación y levantamiento de datos realizada para construir las biografías de las editoras que comprende este tramo, muestra que un número importante de ellas ejercieron la carrera docente, que fue su puerta de entrada a la profesionalización. Es el caso de Delfina Hidalgo, Leonor Urzúa y Juana Adriazola.

Cuando se habla de la profesionalización de las mujeres en la escritura, se establece su capacidad de sostenimiento económico como un indicador de ello. Esto se comenzó a dar en el mundo hispanoamericano a partir de 1850 de forma muy tímida con el auge de la industria literaria por entregas, en España, por ejemplo. Sin embargo, las mujeres que se dedicaban a escribir para vivir sufrían los prejuicios que cuestionaban su moral, en la medida que comenzaban a habitar un espacio de dominio masculino. En este caso tenemos a la española María Martínez Sierra (Fernandez y Ortega 2008: 10) o el caso emblemático de Juana Manuela Gorriti (Batticuore 30-37). Para el caso chileno la investigación biográfica que presentamos de las editoras en estudio muestra que pudieron desarrollar sus empresas por contar con una base económica dada por su condición de herederas ya sea de la fortuna familiar parental o por viudez. De tal forma, sus proyectos editoriales se sostenían más por el compromiso político que significaba su propuesta editorial, que por el rédito económico. Sin embargo, el caso de Juana Adriazola evidencia que el oficio de editora podía convertirse en una labor remunerada para las mujeres (como de hecho lo fue para el siglo XX). La trayectoria de Adriazola muestra que fundó y mantuvo una serie de periódicos en su localidad que, dadas las características de éstos, le dieron réditos económicos.

Por otra parte, tenemos a escritoras que son definidas como profesionales por su trayectoria de escritura propiamente tal y su calidad de intelectuales. Un caso importante es el de la colombiana Soledad Acosta Samper que fue una de las intelectuales más reconocidas de su tiempo y fue profesional en tanto se relacionó y ejerció como escritora y se alzó como publicista, es decir como una hacedora de opinión pública, formadora de un público (Alzate 2015año). El reconocimiento fue algo que las propias escritoras establecieron como evidencia de su profesionalidad. Se consideraban profesionales de las letras y de esa forma querían ser conocidas (Fletcher 214). En la profesionalización de las escritoras latinoamericanas del siglo XIX un espacio fundamental fue el que se abrió con el desarrollo de la prensa periódica. En ella pudieron ingresar textos diversos en el temprano siglo XIX: poemas, crónicas, novelas, ensayos de género y sobre problemas sociales y políticos. Comenzaron a abrirse secciones exclusivas para la pluma de mujeres. Sin embargo, el mayor impacto lo tuvo el inicio de la prensa de mujeres, es decir aquella hecha por mujeres que reflexiona 
sobre el lugar de las mujeres (Montero 2016). Muchos de los nombres de esas pioneras han quedado olvidados, tanto en las historias de la literatura como en la historia de la prensa. Lo mismo sucede con las editoras, que no son reconocidas como mujeres de opinión en el mundo de las letras y de la cultura impresa.

\section{Trayectorias de las editoras de prensa en Chile a Fin Del SIGLO XIX}

Las editoras que nos ocupan surgen a partir del trabajo sobre historia de la prensa de mujeres en Chile (Montero 2016). Esta investigación develó la presencia de mujeres haciendo más que prensa de y para mujeres, sino incursionando en prensa para público general y levantando diversos proyectos editoriales. Algunas de ellas son conocidas por sus actividades intelectuales y no como editoras, por lo tanto, sus biografías se construyeron sobre la base de bibliografía secundaria. De las editoras que estaban completamente olvidadas, sus biografías se realizaron a través de investigación histórica documental.

Las biografías han sido una práctica habitual en estudios historiográficos y literarios, estableciendo diversos métodos para la comprensión de fenómenos. Estos van desde posturas tradicionales que rescatan la vida de "grandes hombres", pasando por aquellos que utilizan la biografía para acercase a problemas epocales, o los que sostienen que una figura biografiada condensa un momento histórico particular. Otras posturas plantean que las biografías dan cuenta de fenómenos dinámicos de una época, iluminando lo diverso dentro de un cuadro complejo. En este caso iluminan la función que cumplieron las mujeres en la conformación del campo y el espacio público.

\subsection{Rosario Orrego: la autoformación de una editora literaria}

Ella fue una escritora e intelectual reconocida tanto por sus contemporáneos como por la crítica actual, de hecho, fue nombrada miembro honorario de la Academia de Bellas Letras en 1873. De ella se ha dicho que fue la primera novelista chilena, además de poeta, ensayista, editora y educadora. Como editora, fundó la Revista Valparaíso (Valparaíso, 18731875), publicación que la llevó a ser calificada en su época como la primera periodista de la República (Figueroa 1891). Este último calificativo considerando que el periodismo era reconocido en tanto profesión en la época no por el título de periodista, sino por la práctica de escribir y o producir prensa.

Rosario Orrego nació en Copiapó en 1831, ciudad de gran actividad minera, y por lo tanto una ciudad moderna con un gran desarrollo urbano y cosmopolita. Se supone que fue educada en su hogar, como era la costumbre entre las familias de la clase acomodada a la que pertenecía. Su madre fue Rosario Castañeda y su padre Manuel Orrego, propietarios de empresas mineras. Su primer marido fue Juan José Uribe, con el que se casó en 1843, y quien también estaba vinculado a la minería. Con él tuvo nueve hijos, entre ellos Regina Uribe, primera bachillera de humanidades. Enviudó joven, Juan José murió en 1853. En algún 
momento migró a Valparaíso, aunque no se sabe si aún casada o una vez viuda. Tampoco se conocen las razones de su mudanza, pero suponemos influyeron en su decisión los conflictos civiles desatados en la zona minera en el 51 y el 59. 20 años después, en 1874, se casó en segundas nupcias con el abogado, periodista y escritor Jacinto Chacón (Godoi 17).

En su condición de viuda, a partir de 1858 inició su período de mayor producción escritural. No sabemos si esto sucedió porque el marido le impedía publicar sus escritos, o porque pudo dejar de lado las tareas de cuidado y administración del hogar con la viudez, o ambas. Ese mismo año publicó por primera vez sus poesías en el periódico La Semana (Santiago 1860-1863) dirigido por los hermanos Domingo y Justo Arteaga Alamparte. Estas publicaciones las hizo bajo el seudónimo "Una Madre" que continuó usando en sus colaboraciones en la prensa como la Revista del Pacifico (Valparaíso 1858-1861) y en la Sud-Americana (Valparaíso 1860-1863), medios en los que publicó por primera vez, y por entregas, sus novelas; Alberto el jugadory Los Busca-Vidas, respectivamente, entre 1861-1864. Esto la convirtió en la primera novelista chilena. Por sus poesías logró el reconocimiento de sus contemporáneos, como se puede observar en la siguiente cita de Pedro Pablo Figueroa:

Sin ser ingratos y olvidadizos, no podemos dejar de traer a la memoria el recuerdo de esta ilustre poetisa que encantó en su tiempo con su gracia femenina y su genio. Ninguna de las mujeres de Chile que han cultivado las letras, ha reunido en su talento y en su persona cualidades mayores ni más ejemplares que ella, por sus virtudes de madre y su amor al progreso, por su civismo nacional y su consagración al arte de la prensa (Figueroa 181).

El reconocimiento como mujer de letras e intelectual se puede observar además en la consideración de su obra poética en diversas antologías nacionales y latinoamericanas.

Al echar a andar el proyecto editorial de La Revista de Valparaíso, se convirtió en la primera editora literaria de Chile. Esto representa un perfil de mujeres intelectuales que publicaron revistas literarias en concordancia con un proyecto cultural de desarrollo de la literatura nacional en un momento determinado. Al convertirse en editora de una revista literaria, combinó su calidad como escritora con la estrategia de posicionamiento. Ella sacó partido de las posibilidades que entregaba la prensa y específicamente la flexibilidad de los medios periódicos para materializar una política de desarrollo cultural que incluyera a las mujeres. Esto le permitió ser "una de las primeras mujeres chilenas en devenir en productora cultural y reclamar el derecho a la instrucción del sexo femenino, así como para la contribución que realizó al desarrollo y consolidación de las letras nacionales" (Contreras 104). De hecho, previo a su acción editorial, tuvo experiencias de inserción al campo cultural como conferencista y sálònier. De esto último nos quedan los comentarios de P.P. Figueroa:

Su genial vehemencia la impelió a fundar en Chile la literatura de salón, que ha sido tan peculiar de la Francia. Sus salones se convirtieron en academias donde acudía el genio en ciernes, como el aquilatado, a revelar sus preciosos dones (...) Se presentó 
llena de encantos a la sociedad y a la juventud, y se transformó en el ideal de la poesía caracterizada por una mujer de genio y belleza inconmensurable (Figueroa 182).

Joyce Contreras la destaca como conferencista en una de las sesiones de la Academia de Bellas Letras, dirigida por José Victorino Lastarria, que también la incorporó como socia honoraria:

No se trata de un texto leído en cualquier lugar, por ejemplo, en una tertulia, sino que es presentado en una de las instituciones culturales más prestigiosas -junto con la Universidad de Chile del momento: La Academia de Bellas Letras. Esto nos habla preliminarmente de dos cosas: la primera, de cómo hacia 1870, luego de casi quince años dedicados al trabajo intelectual, Rosario Orrego había devenido en una figura investida de cierta legitimidad simbólica, algo que aun resulta anómalo para su época; en segundo lugar nos muestra que la autora ocupó dicho espacio de consagración intelectual masculino para introducir, desde su posición de agente cultural genéricamente subalterna, la demanda que reivindica la instrucción femenina (Contreras 70).

Rosario Orrego fundó La Revista de Valparaíso (Valparaíso, 1873-1875) con la que inauguró una nueva relación de autoría femenina con el espacio público, en lo que Carol Arcos (2014) denomina la autoría descubierta. En esa revista literaria publicó sus tres primeras novelas: Alberto el jugador, Teresa y Los Busca-Vidas. Lo hizo por entregas, a modo de folletín, que era un formato propio de la prensa moderna. Estos gestos: el de publicar por entregas, a una mujer, que era ella misma (la primera novelista nacional femenina), denota esa plasticidad de la que hablamos en la definición de editora. Por una parte, decidió fundar una revista que le permitiera desarrollar su proyecto cultural. Esto lo hizo considerando las normas de generificación de las prácticas de lectura y escritura de la época (Poblete 2006). De manera que utilizó el formato de la prensa periódica, que por su flexibilidad permitía que las mujeres pudieran manejarlo y apropiarlo para intervenir en el campo cultural de forma no violenta, o que permitía que la transgresión fuera leve, con un margen de maniobra (Montero 2016). Se acomodó a las condiciones de posibilidad del campo cultural y espacio público que le tocó habitar y disputar. Con ello pudo emitir opinión y concretamente plantear un proyecto político/cultural salvando las normas que pudieran poner en duda su moral por estar ocupando un lugar que no era apropiado para una mujer.

La decisión editorial de publicar una novela por entregas no era una novedad, sí lo era el género sexual de la autora de la obra seleccionada para aparecer en las páginas de su revista. Que en este caso era ella misma. Desde su lugar de editora decidió publicar a una mujer para insertarla (insertarse) en el campo cultural e intelectual. Publicarse a sí misma le valió saltarse la regla de la autorización, es decir aquella en que las mujeres para publicar una obra debían ser avaladas o autorizadas por un hombre (Arcos 2014). Esto último lo hizo posteriormente, cuando publicó de manera íntegra en formato libro 
su novela Alberto el Jugador. Novela de Costumbres (1861), que prologó Ricardo Palma. Sin embargo, la transgresión ya había sido hecha: publicó su novela, la que la convirtió en la primera novelista de Chile, sin autorización y en una revista que era de su responsabilidad y, por lo tanto, donde ella tomó esa decisión.

\subsection{Delfina Hidalgo y Ruth Wetherby: el desarrollo como editoras de medios de propaganda religiosa}

Delfina Hidalgo y Ruth Wetherby fueron mujeres que editaron medios de propaganda religiosa. Delfina Hidalgo fue redactora de El Predicador (Valparaíso, 18861887) y directora de La Aurora (Valparaíso, 1888-1890). Por su parte Ruth Wetherbyd está relacionada con El Intransigente (Valparaíso, 1888-1891) y Propaganda de la Reforma Social (Valparaíso, 1891-1905).

Ambas mujeres desarrollaron sus actividades como editoras en la ciudad de Valparaíso, en momentos del auge económico. El fin del siglo XIX el puerto principal de Chile se convirtió en un polo de atracción. Miles de personas llegaron a ocuparse en las actividades portuarias, comerciales e industriales, no sólo desde todos los rincones del país, sino también desde el extranjero. Valparaíso se alzó como una urbe moderna, cosmopolita y multicultural. Por lo tanto, no es casual que los primeros periódicos de propaganda religiosa no católica se publicaran allí (considerando la tradición católica de Chile), lo mismo que muchas de las editoras que nos convocan llegaran a Valparaíso y desarrollaran sus proyectos en la ciudad. Una ciudad que se abría a todo y todos.

El caso de Ruth Wetherby y Defina Hidalgo corresponde al de mujeres que formaron parte de redes familiares que tenían una vocación por el servicio público y la propagación de una determinada fe. Ruth Wetherby fue hija de Rosario Vivencio (1849-1919) una de los cuatro chilenos que figuran como miembros de la primera iglesia evangélica fundada en Santiago en 1868 (Mc Lean 35). Su padre fue N. J. Wetherby (?- 1912) médico inmigrante estadounidense que llegó a Chile como evangelista. La familia se instaló en Valparaíso hacia 1870 y desarrolló una red familiar, comercial y cultural relacionada con la promoción de las iglesias evangélicas, que era el proyecto del Sr. Wetherby. Fundaron la Sociedad Bíblica de Valparaíso, La Sociedad de la Abstinencia (1878) y varias logias o "sociedades de temperancia" que tenían el objetivo de erradicar el alcoholismo. Fue en éstas logias donde actuaron las hijas de la familia. Datos de 1888 muestran que Ruth fue la vicepresidenta de la logia "Arturo Prat" y Rebeca prosecretaria de la misma. Hacia 1892 Rebeca fue prosecretaria de la logia "Esmeralda" y Ruth capellán. Como parte del plan de N. J. Wetherby comprometido con la regeneración social, fundó el primer periódico propagador de la temperancia que se publicó en América Latina (Pacheco): El Intransigente (Valparaíso 1888-1891) y su continuador Propaganda de la Reforma Social (Valparaíso 1891-1905). Esta actividad se vio facilitada por ser dueño de una imprenta y librería (Prado 580). En ella se dedicaba a la publicación de periódicos y libros de lectura en general, lo que lo ha llevado a ser considerado como parte del grupo de los destacados empresarios de la prensa (Ugarte). 
Ruth Wetherby fue la hija que, a pesar que en los periódicos no se la menciona como la directora de ellos, se encargó de las labores de dirección y edición. Todo esto ha sido corroborado por bibliografía secundaria, en la que se establece a Ruth Wetherby como la responsable de los medios (Ugarte 284). En concreto, una nota de Propaganda de la reforma Social Valparaiso, periódico también asociado a Wetherby es esclarecedora de la situación. Esta nota confirma a Ruth como la persona a cargo de las decisiones del medio. En plena crisis política de 1891, decidió cerrar el periódico:

El periódico que veía la luz en este Puerto titulado El Intransigente dejó de publicarse en el mes de Julio pasado. Su propietaria no teniendo en aquella época garantía alguna para poder manifestar sus ideas con toda la franqueza y libertad deseada, creyó más prudente suspender su publicación, hasta ver despejado el horizonte político para poder continuar en la noble tarea que había emprendido. (s/a)

Con los pocos datos de la biografía de Ruth Wetherby con los que contamos, podemos hacernos una idea del perfil que representa. Por una parte, constatar la importancia de una familia que animó la educación de las hijas. Y como éstas, estimuladas por sus padres, se transformaron en personajes públicos, llegando a desarrollar actividades directivas en organizaciones sociales. De tal forma, Ruth Wetherby desarrolló su labor de editora como otra actividad más dentro de una serie de acciones que formaban parte de un proyecto mayor. Proyecto que no sólo era familiar o de una comunidad, sino que personal, en la medida que le permitió desarrollarse como persona y actor político y social. Como editora navegó en la crisis política, tomando la decisión más difícil para una persona comprometida con la libertad de expresión y con confianza en la labor y poder de la palabra pública: cerrar el medio que dirigía. Esto no implicó su silencio, ya que siguió con sus labores públicas y posteriormente fundó un nuevo medio.

De una manera diferente Delfina Hidalgo también asumió la edición de periódicos de propaganda religiosa por pertenecer a una red familiar. Esta vez asociada a su marido, Alberto Morán Rojas (1857-1930), quien fue el primer pastor presbiteriano chileno en una congregación del habla castellana. Este a su vez era hijo de Buenaventura Morán profesor ligado a la tipografía y el periodismo, fundador el periódico El Taller (Santiago, 1879) y el periódico satírico El Padre Cobos (Santiago, 1875-1894). Como parte de esta red, Delfina Hidalgo fue redactora en Valparaíso de El Predicador (Valparaíso, 1886-1887) periódico de propaganda cristiana, al igual que el que fundó: La Aurora (Valparaíso, 1888-1890).

Sin embargo, Delfina Hidalgo llegó a la edición luego de consolidarse como una mujer profesional de las letras. Nativa de la ciudad de Copiapó donde nació en 1862, fue educada en distintos colegios de la ciudad. A los catorce años, en 1876, inició sus colaboraciones en medios con la publicación de su primer poema en el periódico $E l$ Constituyente de la ciudad de Copiapó. Durante su carrera colaboró en: El Atacama, La voz del Estudiante, La Revista Literaria, La Mujer, el Heraldo, El Imparcial, el Nuevo Ferrocarril, El Trabajo, Las Novedades. Viviendo en Antofagasta publicó su primer libro en 1880 Ensayos 
Poéticos (Antofagasta: Imprenta de El Pueblo Chileno), lo que le valió el reconocimiento de la crítica. Por esa época también escribió los ensayos: "Dos clases de poesías", "Ciencias e Inteligencia" y los dramas "Dime con quién andas..." y "La Instrucción de la Mujer". Su obra le valió el premio de la Academia de Ensayos Literarios (Eliz 267) y el reconocimiento por participar en el Certamen Varela en 1887. Allí se presentó con el pseudónimo Violeta en el segundo tema del concurso "Poesía Líricas" y fue reconocida como "la mejor colección de (doce a quince) composiciones inéditas de poesía del jénero (sic) sugestivo o insinuante, de que es tipo el poeta español Gustavo A. Bequer, premio 500 pesos."2 Ejemplo del reconocimiento de la crítica fue cómo Pedro Pablo Figueroa la identificó dentro de una nueva generación de escritores:

Una revolución literaria de trascendentales efectos se opera en la juventud haciendo surgir de la oscuridad y del silencio nombres ignorados, inteligencias desconocidas y obras notables que vienen a enriquecer la literatura patria.

Pertenecen á este movimiento intelectual los jóvenes poetas y prosistas Pablo Garriga, Santiago Escuti y Orrego, Eduardo Poirier, Alberto del Solar, Jorge Huneeus Gana, Leonardo Eliz, Raimundo del R. Valenzuela, Horacio Lara, Luis Arrieta Cañas, Salvador Soto, Delfina María Hidalgo, Pedro N. Cruz y otros no menos perseverantes é instruidos. Periódicamente aparecen en la arena literaria sobresalientes inteligencias, exhibiendo obras de verdadero mérito que nos encantan por su belleza y nos ejemplarizan con su enseñanza (Figueroa 82).

Su profesionalización como mujer de letras y editora tuvo un paso previo fundamental como educadora. Labor que inició antes de cumplir 18 años y que desempeñó en las distintas ciudades en las que vivió: Copiapó, Antofagasta y Valparaíso. Ejerció como profesora en el Liceo de nińas (Copiapó, 1877), la Escuela Rafael Valdés y fue directora de distintas instituciones educativas a lo largo de su vida, entre ellas el Liceo de San Fernando (1883-1884).

Delfina Hidalgo, al igual que Ruth Wetherby son una muestra de que la acción editorial fue una labor más a lo largo de las carreras de mujeres que tenían una acción pública con un proyecto particular. El caso de Hidalgo, nos muestra además una mujer profesional tanto de la educación como de las letras, lo que le dio la base para desempeñarse en su labor de editora.

\subsection{Lucrecia Undurraga y Leonor Urzúa: Activistas y editoras feministas}

Lucrecia Undurraga y Leonor Urzúa desarrollaron medios de prensa con una línea editorial feminista. Entendemos como feminista la idea que plantea Asunción Lavrín para la época, que remite a toda acción femenina en el ámbito público, en particular aquellas

${ }^{2}$ El Programa del Certamen fue publicado en la "Libertad electoral" del 28 de junio de 1887. 
que tenían un tono progresista y que consideraban la instrucción femenina una demanda fundamental para el desarrollo de las mujeres. Lucrecia Undurraga editó La Brisa de Chile, San Felipe 1875-1876 y La Mujer. Periódico semanal: Historia, politica, literatura, artes y localidad, Santiago 1877, y Leonor Urzúa La Mujer, Curicó 1897 y Almanaque Literario de la Mujer, Curicó 1899).

Ambas pertenecieron a familias que les permitieron desarrollar sus inquietudes políticas e intelectuales. Lucrecia Undurraga nativa de Illapel, nació en 1841 en una familia de renombre local dedicada a la agricultura. Se casó con José Manuel Somarriva, empresario vinculado a la minería, con quien tuvo un hijo y enviudó joven. Esta situación le permitió heredar una estancia que según Carla Ulloa la dejó en una posición económica cómoda, aunque ello no la incluiría dentro de los espacios y relaciones de la aristocracia de la época (Ulloa 112). De niña fue enviada a estudiar al colegio "Valparaíso" dirigido por la señora Josefa Cabezón, quien influyó fuertemente en el pensamiento de Lucrecia en lo referido al compromiso social para el desarrollo de las mujeres: allí vio cómo la directora recibía gratuitamente a muchachas modestas.

Su faceta intelectual la desarrolló plenamente una vez viuda, cuando se mudó a Valparaíso y Santiago. Como otras profesionales de la letra, inició su actividad como escritora colaborando en medios como La Revista del Pacifico (Valparaíso, 1858-1861), La Sud Americana (Valparaíso, 1860-1863) y La Brisa de Chile (San Felipe, 1875-1876). Fue reconocida como escritora por sus pares varones y mujeres. Al igual que Rosario Orrego presentó un texto en la Academia de Bellas Artes, fue mencionada por la colombiana Soledad Acosta de Samper en su libro La mujer en la sociedad moderna (Acosta de Samper) en el apartado dedicado a las escritoras chilenas:

Como de todo ha de haber en América, Chile cuenta también con una escritora socialista, la señora Lucrecia Undurraga de Somarriva. Tanto en una revista que fundó, como en libros y publicaciones que ha hecho, se esfuerza en sostener valientemente sus ideas de avanzada y abrir nuevos horizontes a la mujer proletaria (Acosta de Samper 212-214).

También fue incluida en el Diccionario Biográfico de PP Figueroa (Figueroa), por lo que Leonor Urzúa, otra de las editoras feministas, la reconoce como escritora y educadora feminista dentro de una genealogía de mujeres chilenas:

Tenemos poetisas i escritoras distinguidas, como Mercedes M. del Solar, Rosario Orrego de Chacón, Lucrecia Undurraga de Somarriva, Amelia Solar del Claro, Quiteria Varas Marín, Mariana Cox de Stuven, Inés de Echeverría de Larraín, Amelia Errázuriz de Subercaseaux, Delfina María Hidalgo, Teresa Prats de Sarratea, Rosa Caballero de Altavilla, Celia G. de Fortin, Celia Soto Glen, Rosa Nercasseau i Moran, Blanca Vanini, i otras cuyos nombres se nos escapan por el momento. (...) ¡Que nuestra humilde voz sea eco que llegue hasta nuestras generaciones futuras! (Urzúa) 
Como editora Lucrecia Undurraga tomó decisiones que dan cuenta de ser una mujer con opinión política. Si bien no se puede asegurar que fue parte del grupo editorial de La Brisa de Chile, Carla Ulloa (2017) la asocia a ésta publicación por su constante colaboración en ella, con textos comprometidos. En uno de ellos se pregunta por cuál debía ser el objetivo de la prensa, a lo que responde que no otro que el debate político. Carla Ulloa analiza el texto "A los señores editores de la Brisa de Chile", afirmando que Undurraga se hizo cargo de la tensión existente entre literatura y política que estaba en la base del desarrollo de la prensa de la época. De tal forma, la apuesta de Undurraga fue la politización de los contenidos, dejando de lado los referidos al desarrollo de una literatura femenina. La máxima de Undurraga sería que la política llevaría a la ilustración de las mujeres. Según Ulloa, esas ideas, las del desarrollo de la discusión política, estuvieron en la base del proyecto de La Mujer (Santiago, 1877) (121-122).

En La Mujer tomó todas las desiciones que la instalaron como la mujer socialista, liberal y feminista con las que se hizo conocida. Defendió la instrucción de las mujeres a través de textos que analizaban la condicion femenina de la época. Esto supuso una acción de mediación, traduciendo para un publico específico, las mujeres, los derechos que ella creía era necesario que debían demandar políticamente. Como gestora, usó La Mujer para articular una red de mujeres intelectuales que encontraron en ese periódico un lugar de reunión pública, discusión y expresión. Es así como se encuentran en sus páginas una larga lista de mujeres que escribieron desde diversos tipos de textos y géneros literarios. Las secciones del periódico son la expresión de desiciones editoriales que responden a un proyecto político: hacer presente la voz de las mujeres en el espacio público, inclyendo esos trabajos invisibilizados como la traducción, géneros nunca asociados a la pluma femenina, como el análsis político, y evitar el reforzamiento del estereotipo femenino tradicional excluyendo todo lo relacionado con la moda.

Por su parte, Leonor Urzúa nos muestra una trayectoria de compromiso político con la defensa de desarrollo de las mujeres que expresó en diversos proyectos, incluyendo la edición de periódicos. Ella fue una entre 18 hemanos, hijos de una familia oligarca compuesta por Blas Urzúa y Daniela Cruzat y Céspedes. Ambos descendientes de españoles asentados en Curicó. Nació en 1868 y fue educada según los principios liberales de su padre: en igualdad de condiciones hombres y mujeres tarea en la que invirtió parte de su fortuna. Esta educación fue provista en casa y produjo una camada de intelectuales, periodistas y escritores (Eliz, "Prólogo" 8) como Pedro Nolasco, periodista y abogado; Domingo Urzúa, periodista y profesor; Elías Urzúa, uno de los fundadores del partido demócrata en Curicó. Los principios de igualdad con las que el padre formó a sus hijos se evidencia en las actividades desarrolladas por las hijas. Todas se dedicaron a la enseñaza y la escritura. Deyanira Urzúa fue poeta, dramaturga, pionera de la literatura infantil en Chile y educadora. Ejerció como profesora en distintas ciudades del país e incluso fundó el 1937 el Colegio Urzúa Cruzat en Santiago. Susana Urzúa también fue educadora vinculada a la música. Compuso piezas, fue locutora radial y autora del libro Influencia Moral y psicológica de la Música y el Canto (Pavés 99). 
Este contexto familiar nos ayuda a comprender la trayectoria de Leonor Urzúa, ya que podemos observar el ambiente en el que creció, las influencias que recibió, las prácticas en las que fue formada, como la lectura y las redes con las que contaba para desarrollar su actividad pública. La podemos definir como feminista, escritora, maestra, gestora cultural y editora.

A pesar de que no recibió educación formal y por lo tanto no tuvo título alguno, trabajó toda su vida como educadora en distintos cargos: como profesora y directora de colegios. En 1892, junto con sus hermanas Genoveva, Susana y Deyanira, invirtió parte de su fortuna en fundar el primer Liceo de señoritas de Curicó. La visión crítica y la apuesta política de las hemanas Urzúa no está sólo en el hecho de hacerse cargo de la necesidad de educación de las mujeres, sino en la calidad de ésta. Más allá de replicar currículums marcados por normas de género que remitín la educación femenina a labores de hogar, ellas implementaron ramos de biología e higiene, moral y urbanidad, italiano y declamación. Su labor en educación la realizó complemetando su acción como gestora cultural: fundó en 1896 la Estudiantina Curicó y la Academia Literaria Mercedes Marín del Solar. En ésta última estimuló el desarrollo intelectual de las mujeres de la ciudad de Curicó, ya que no sólo estaba abierta a las estudiantes del liceo, sino que a todas las mujeres de la ciudad. Bajo la institucionalidad de la Academia creó un espacio de encuentro, discusión y organización femenina a través de reuniones mensuales donde se desarrollaban conferencias sobre literatura, historia, sociología, entre otros. En ellas se puede ver la función de mediación que cumplió junto con sus hermanas, labor que reforzó con la publicación de La Mujer (Curicó, 1897-1898), vocero de la Académia Literaria Mercedes Marín del Solar.

Las desiciones editoriales de Leonor Urzúa dan cuenta del proyecto feminista que llevaba adelante, y que en La Mujer cobran cuerpo en toda su materialidad. Para imprimir la revista "compró una pequeña prensa y formó e incluso hizo aprender tipografía a dos "niñas pobres", para sí de principio a fin su publicación fuera fruto del trabajo femenino" (Eliz, "Prólogo" 10). Este fenómeno de la impresión doméstica de medios ha sido estudiada en otros países, para Chile, es la primera noticia que tenemos de ello. Esto implica que tuvo que desarrollar unas habilidades manuales para manejar las máquinas o para supervisar su manejo que la capacitaron como profesional de la prensa. En este caso, nos queda más claro que tuvo que desarrollar competencias en diagramación, corrección de pruebas y otras labores asociadas a la imprenta. Sin dejar de lado la capacidad de administrar el personal y de formarlo en sus labores.

En relación con los contenidos, La Mujer publicó textos de las socias de la Academia, evidenciado el estímulo al desarrollo de las letras femeninas locales que estaba en el proyecto de Urzúa. También incluía artículos de análisis que podemos calificar como ensayos de género, propios de la prensa de mujeres (Montero 2016). Muchos de ellos de autoría de la propia editora y que materializan no sólo un discurso feminista, sino que expresan cómo la prensa fue un espacio escogido y utilizado por las mujeres para poner su voz en el espacio público. De tal forma, la desición editorial no sólo tiene que ver con formatos y contenidos, sino también con elegir un medio que permitiera lograr objetivos. 


\subsection{Juana Adriazola y Celeste Lasabe: Profesionales de prensa comercial}

Juana Adriazola y Celeste Lasabe desarrollaron medios para su circulación mediante la compra y la venta. No podemos asegurar que fue una actividad comercial que permitió la subsistencia de éstas editoras. Sin embargo, definitivamente desarrollaron estas empresas no sólo con el objetivo de exponer un proyecto cultural o político, invirtiendo todo en ello, sino tomando las ventajas de la venta de un producto que además tenía un contenido, donde ellas eran las mediadoras para el púbico.

Juana Adriazola editó El Correo (Osorno, 1880-1887), El Rio Bueno (Río Bueno,1887-1888), La Pampa (Río Bueno, 1889-1890), La Paz (Río Bueno, 1891-1894), con ello se hizo una profesional de la prensa y que seguramente logró su sustento mediante ella. Nació en Valdivia en 1858 y fue hija de Miguel de la Cruz Adriazola y Emilia Uribe, ambos pertenecientes a familias espańolas asentadas en Valdivia con fines administrativos. Se educó en un instituto superior femenino de su ciudad, donde fue nombrada ayudante. Ese cargo lo ejerció por seis años, logrando una entrada al mundo profesional a través de la enseñanza. Al momento de casarse abandonó esa labor, sin embargo sus deberes de esposa del periodista José María Casas, con quien se casó en 1876, no impidieron que iniciara una carrera en el mundo de la prensa.

José María de Casas también provenía de una familia terrateniente de Valdivia. Tal vez esta condición le permitió dedicarse al periodismo, labor que desempeñó fundando periódicos en distintas ciudades del sur de Chile. Cuando Juana y José María se establecieron en la localidad de Río Bueno, no existían periódicos locales, por lo que la pareja inició la labor de fundar los primeros de la zona: La Pampa y Río Bueno. Esto fue posible gracias a que desde la mitad del siglo XIX existía una imprenta en la ciudad. Viuda en 1891, Juana Adriazola asumió la labor completa de continuar con los periódicos, además de fundar otro más: $\mathrm{La} \mathrm{Paz}$. A través de él Adriazola materializó la decisión de publicar no sólo un periódico noticioso "de formato pequeño, de selecta impresión y redacción. Tiene todas las secciones, aunque reducidas, de todos los grandes periódicos y su material político, literario, noticioso y de avisos, es muy superior a los demás diarios de los departamentos del sur" (Figueroa 114), sino que representó una posición política en medio de un conflicto político. La Paz fue de marcada tendencia liberal y defendió el liberalismo representado por las posiciones pro-balmacedistas en medio de la Guerra Civil de 1891.

Las decisiones de Juana Adriazola las inició junto con su marido al desarrollar prensa en un lugar donde no existía. Esto equivalía llevar la modernidad a una localidad, acercarla al mundo, crear un público a través del desarrollo de prácticas de lectura. Llevar la urbe introduciendo un nuevo dispositivo de modernidad y comunicación. Eso explica el tipo de medio que desarrolla: noticioso, pero con un poco de todo. También su forma de circulación: comercial, ya que debía llegar a todo el mundo. Finalmente, la decisión de representar a un bando político, pero sin desarrollar la prensa de barricada característica de principio del siglo XIX, sino un medio moderno, que expresaba opinión frente a la contingencia sin comprometer la vocación del periodismo que es informar. 
Celeste Lasabe (Francia ¿? - Santiago 1927) fue hija de una familia tradicional y católica de la pequeña nobleza de la región de Tarbes en Francia. Vivió en modestos castillos, "gente cuidadosa, respetable y económica. Guardiana de bienes, tradiciones y privilegios" (Cruz-Coke 56). Celeste Lasabe fue una mujer ilustrada, que leía literatura y la prensa diaria tanto local como extranjera. Cuidó ciertas formas aristocráticas como la conservación de un patrimonio en joyas, obras de arte y mobiliario francés. Además del respeto por ciertos valores monárquicos, como evitar celebrar el 14 de julio como aniversario de Francia ${ }^{3}$. Sin embargo, fue una mujer de avanzada, respecto de la consideración de las capacidades de desarrollo de las mujeres en la literatura y el arte, ideas que ella misma encarnó como editora.

Celeste Lassabe, llegó al puerto de Valparaíso acompañando a su marido Philips Dubiau, con quien tuvo una hija, Marie Lucíe Dubiau. Él fue un exitoso empresario francés, sin embargo falleció al poco tiempo de llegar a Chile. Viuda, con una hija y en un país extraño, pero heredera de una fortuna, optó por quedase en Chile. Se instaló definitivamente en Valparaíso porque era una sociedad abierta que le permitía desarrollar sus inquietudes intelectuales (Cruz-Coke 56). Pasados unos años, se casó con el abogado y escritor Ricardo Cruz-Coke $e^{4}$ quien se dedicó a la labor periodística. Instalados en el Cerro Alegre, la pareja se convirtió en parte de la elite intelectual de la ciudad. Celeste pudo desplegar la tradición cultural francesa del salón, en las veladas que organizaba junto a su marido para intelectuales y artistas que pasaban por la ciudad puerto (Cruz-Coke 58).

Esta es la mujer que fundó y dirigió La Familia. Publicación quincenal Ilustrada de literatura, ciencias artes y modas, que salió en Santiago entre 1890 y 1893, con lo que se inauguró en Chile la revista ilustrada, un dispositivo moderno. En ella integró elementos posibilitados por la modernización: la publicación de gravados, reproducción de imágenes, publicidad, junto con una diversidad de tipos de textos y temas (Arcos 2016). Como editora, tomó decisiones fundamentales que la convirtieron en una interventora y mediadora en el espacio público. Primero que nada se conformó como empresaria, ya que desarrolló un producto para un mercado en formación. Aprovechó las condiciones de posibilidad de una emergente economía capitalista, donde vislumbró un público al que vender un producto nuevo: una revista para las mujeres de elite que debía lidiar con la sociabilidad de la emergente cultura urbana (Montero, "Trocando agujas por la pluma: las pioneras de la prensa de y para mujeres en Chile 1850-1890"). Parte de sus decisiones de funcionar como empresa fue establecer estrategias para alcanzar el mayor número de lectores posible. De tal forma se valió del agente de prensa para la distribución, posible sólo por el moderno sistema de transporte y correo que recién se instalaba en el país. Introdujo publicidad, lo que, junto a tiradas de 10.000 números, evidencian la vocación comercial del periódico.

\footnotetext{
3 "Entre otros motivos porque la revolución había guillotinado a su tatarabuelo Lucien Lassabe, médico de cámara de Luis XVI" (Marta Cruz-Coke 58).

${ }^{4}$ Sus publicaciones literarias son: Corazón de León: novela santiaguina, Santiago: Cervantes, 1890, y Guelfos y gibelinos, Santiago: Cervantes, 1894.
} 
Como mediadora, Celeste Lasabe entregó claves para sobrevivir en una sociedad moderna y urbana, que suponía nuevas interacciones ciudadanas y letradas, en las que nadie tenía experiencia. Esto se materializó en las secciones que dan cuenta del carácter magazinesco de la revista, que mezclaba consejos hogareños, notas científicas, textos literarios, entre otros. Sin embargo, no podemos quedarnos sólo con este aspecto de la publicación que podría ser considerado liviano. Ya que Celeste Lasabe decidió incorporar análisis políticos. Demostró una gran capacidad para gestionar el formato revista y poder discutir ideas sin que generaran acusaciones de transgresión (porque era una mujer la editora) o por ser asociada a alguno de los bandos políticos en conflicto, Celeste Lasabe publicó estos análisis valiéndose de la carta. Este tipo de texto íntimo, asociado a lo femenino lo gestionó de forma que en medio de una crisis política entre conservadores y liberales, Lasabe criticó a la clase política en su sección "Cartas Japonesas". Estas eran unas cartas que enviaba un tal Conde Tchi, hermano de un diplomático japonés, a quien reportaba sus impresiones sobre el funcionamiento del Estado, la sociedad y la cultura. Como extranjero, no comprometía su integridad, y Lasabe, como editora, lograba enviar su mensaje.

Finalmente, al igual que Rosario Orrego, Celeste Lasabe también decidió publicar su primera novela en su propia revista. Con ello, se saltó la autorización necesaria para ingresar al campo literario, y además las posibles trabas para la publicación de un libro en editorial.

\section{Conclusiones}

Con este trabajo se devela la presencia de mujeres antes no consideradas en la historia de la cultura impresa del país, rescatando sus trayectorias para establecer cómo se hicieron profesionales en tanto editoras de medios. Las trayectorias biográficas nos permiten confirmar algunos hallazgos de otras investigaciones sobre la pertenencia de estas mujeres a las elites económicas, afirmando que no necesariamente formaron parte de grupos de poder, que les aseguraba cierta circulación y/o solvencia económica. Sino más bien que pertenecieron a una elite intelectual y que a través de esas redes pudieron desarrollar sus empresas.

Respecto de la profesionalización, podemos observar que todas muestran una especialización en el oficio de escribir y que, en tanto editoras de medios, usaron esa especialización para diversos objetivos dependiendo de tipo de prensa que desarrollaron. Además, que la edición fue una de entre varias formas de entrada al mundo profesional, ya que muchas lo hicieron a través de la docencia, aunque no fue el único camino. Lo que sin duda encontramos es que hubo algunas mujeres que lograron dedicarse completamente a la edición de medios de prensa y que suponemos lograron vivir de esta actividad, como fue el caso de Juana Adriazola. 


\section{Obras CiTADAS}

Acosta de Samper, Soledad. 1893. La mujer en la sociedad moderna. Paris: Garnier Hermanos.

Alzate, Carolina. 2015. Soledad Acosta de Samper y el discurso letrado de género, 1853 a 1881. Madrid: Iberomaricana.

Anónimo. 1891. Nota editorial. Propaganda de la reforma Social 1.

Arcos, Carol. 2014. Autorias femeninas fundacionales: escritoras chilenas y brasileñas del siglo XIX (1840-1890). Tesis para optar al grado de Doctora en Estudios Latinoamericanos. Santiago: Universidad de Chile.

2010. Novelas-folletín y la autoría femenina en la segunda mitad del siglo XIX en Chile. Revista Chilena de Literatura 76: 27-42.

Batticuore, Graciela. 1994. "Historias cosidas, el oficio de escribir". En Lea Fletcher, Mujeres y cultura en la Argentina del siglo XIX. Buenos Aires: Feminaria, 1994. . 2005. La Mujer Romántica. Buenos Aires: Edhasa, 2005. Impreso.

Brown, John. 2018. The History of the Modern Career: An Introduction. En Brown, John, Marco H.D. Van Leeuwen y David Mitch, Introduccion to the Study of Careers and their History. Holanda: IISG.

Brunner, José y Gonzalo Catalán. 1985. Cinco estudios sobre cultura. Santiago: FLACSO.

Contreras, Joyce. 2017. Las transformaciones del campo cultural a mediados del siglo XIX y el surgimiento de una escritora moderna: Rosario Orrego Uribe. Contreras, Joyce, Damaris Landeros y Carla Ulloa, eds., Escritoras chilenas del XIX. Su incorporación pionera a la esfera pública y al campo cultural. Santiago: RIL.

Cruz-Coke, Marta. 2015. Eduardo Cruz-Coke. Testimonios. Santiago: Fundación Procultura.

Darrigrandi, Claudia. 2014. Huellas de la ciudad: figuras urbanas en Buenos Aires y Santiago de Chile, 1880-1935. Santiago: Cuarto Propio.

Egaña, María, Cecilia Salinas y Iván Núñez. 2000. Feminización y primera profesionalización del trabajo docente en las escuelas primarias, 1860-1930. Pensamiento Educativo 26.

Eliz, Leonardo. 1889. Musas Chilenas 1560-1889. Siluetas líricas y biográficas sobre los más distinguidos poetas nacionales. Santiago: La Unión.

Eliz, Leonardo. 1923. Prólogo. En Urzúa, Leonor, Cuentos Chilenos. Santiago: La Ilustración.

Falabella, Soledad. 2012. Modernidad literaria y la entrada de las mujeres a la esfera pública en los discursos. Revista Chilena de Literatura 82.

Fernandez, Pura y Marie-Linda Ortega. 2008. La mujer de letras o la letra herida. Discursos y representaciones sobre la mujer escritora del siglo XIX. Madrid: Consejo Superior de Investigaciones Científicas.

Figueroa, Pedro. 1891. Diccionario Biográfico Nacional (1550-1892). Santiago: El Correo.

Fletcher, Lea. 2004. La profesionalización de la escritora y de sus protagonistas. Argentina, 1900-1919. Revista Iberoamericana 70, 206.

Godoi, Osvaldo. 2016. Apuntes para la biografía de una madre. Godoi, Osvalvo, Catalina Zamora y Ricardo Tapia. Rosario Orrego 1831-1879. Obra reunida, estudio y compi- 
lación. Copiapó: Alicanto Azul.

González, Alba. 2013. Domesticar la escritura. Profesionalización y moral burguesa en la obra pedagógica de María del Pilar Sinués (1835-1893). UNED 1: 51-99.

González-Leandri, Ricardo. 2006. Campos e imaginarios profesionales en América Latina. Renovación y estudios de casos. Anuario IEHS 21.

Irigaray, Luce. 1985. El cuerpo a cuerpo con la madre. Barcelona: Lasal.

Mc Lean, J. H. 1932. Historia de la iglesia presbiteriana en Chile. Santiago: S/E.

Montero, Claudia. 2016. Trocando agujas por la pluma: las pioneras de la prensa de y para mujeres en Chile 1850-1890. Meridional 7: 55-81.

. 2018. Y También hicieron periódicos. Cien años de prensa de mujeres en Chile 1850-1950. Santiago: Hueders.

Pacheco, Waldo. 2004. Breve cronología histórica del cristianismo protestante en Chile (1572-1940). Colección de estudios evangélicos 6.

Pavés, Francisco. 1943. Desarrollo intelectual de Curicó. Curicó: La Prensa.

Poblete, Juan. 2006. Cambio cultural y lectura de periódicos en el siglo XIX en América latina». Revista Iberoamericana LXXII: 1-15. . 2003. Literatura Chilena del siglo XIX: entre públicos, lectores y figuras autoriales. Santiago: Cuarto Propio.

Prado, Alberto. 1903. Única guia general de Chile. Santiago: Centro Editorial de Alberto Prado Martínez.

Pratt, Mary Louise. 1998. Las mujeres y el imaginario nacional en el siglo XIX. Revista de Critica Literaria Latinoamericana 19, 38.

Rama, Ángel. 1998. La ciudad letrada. Montevideo: Arca.

Ramos, Julio. 2009. Desencuentros de la modernidad en América Latina. Literatura y politica en el siglo XIX. Caracas: El Perro y la Rana.

Subercaseaux, Bernado. 2010. Historia del Libro en Chile. Cuerpo y Alma. Santiago: LOM, 2010.

Subercaseaux, Bernardo. 2008. Historia de las ideas y la cultura en Chile. Santiago: Universitaria, 2008.

Traverso, Ana. 2012. Primeras escritoras en Chile y autorización del oficio literario». Anales de Literatura Chilena: 61-80.

Ugarte, Juan. 1910. Valparaiso 1536-1910. Recopilación histórica Comercial y Social. Valparaíso: Imprenta Minerva.

Ulloa, Carla. 2017. Lucrecia Undurraga y el periodismo liberal de mujeres en el Chile decimonónico: La Brisa de Chile (1875-1876) y La Mujer (1877). En Landeron, Damaris, Joyce Contreras y Carla Ulloa, eds., Escritoras chilenas del XIX. Su incorporación pionera a la esfera pública y al campo cultural. Santiago: RIL.

Urzúa, Leonor. 1912. Flores Incultas. Santiago: Imprenta Santiago. 